\title{
Pneumatic Conveyance of Fiber Assemblies
}

\author{
By Kiyoji Nakamura and Akira Horikawa, Members, TMSJ
}

Faculty of Engineering, Osaka University, Osaka

Based on Journal of the Textile Machinery Society of Japan, Transactions, Vol.23, No.1/2, T24-29 (1970)

\begin{abstract}
The authors have looked into pressure drops in horizontal and vertical straight conveyor pipes and horizontal bent conveyor pipes to obtain basic data for use in designing a pneumatic conveyor system for fiber assemblies which will help forward the modernization of the spinning process.

The concept of friction velocity $u_{f}$ has been applied to the pneumatic conveyance of fiber assemblies to show that the ratio $u / u_{f}$ of mean air velocity $u$ to $u_{f}$ is linearly correlated to the mixing ratio (weight) $m$; and that frictional coefficients $\Lambda$ and $\Lambda^{\prime}$ of fluid for mixed-phase flow (air and fiber assembly) can be obtained.
\end{abstract}

The friction coefficient for a horizontal straight conveyor pipe is as given in eq.(15)

$$
A-\frac{8}{\left(\sqrt{ } / \frac{8}{\lambda}-5.10 \mathrm{~m}\right)^{2}}
$$

The friction coefficient for a vertical straight pipe is as given in eq.(29)

$$
A^{\prime}=\frac{8}{\left(0.95 \sqrt{\left.\frac{8}{\lambda}-12.5 \mathrm{~m}\right)^{2}}\right.}
$$

The coefficient of the pressure drop in a horizontal bent pipe has been calculated by dividing it into $\xi$ for air flow and $\zeta$ which increases as a fiber assembly is added to, and the following equation has been obtained:

$$
\zeta=0.11 \frac{2 \mathrm{R}}{\mathrm{D}}(\mathrm{m} \cdots 0.10) \cdots \cdots \cdots \cdots \cdots \cdots \cdots \cdots \cdots \cdots \cdots \cdots \cdots \cdots \cdots \cdots \cdots \cdots \cdots \cdots \cdots \cdots \cdots \cdots
$$

KEY WORDS: FIBER ASSEMBLIES, COEFFECIENT OF FRICTION, PIPES, TRANSFERRING (MATERIAL)

\section{Introduction}

Planning a pneumatic conveyor system requires a full grasp of the relation between the amount of material transported and the horse-power needed for transportation. This relation resolves itself into the relation between the mixing ratio and the pressure drop. The energy consumed by a pneumatic conveyor is supplied from the pressure energy of the air, which supply constitutes a pressure drop.

The relations between the mixing ratio and the pressure drop as they concern pneumatic conveyors for grains have been looked into, in most cases experimentally, by a host of research workers. Each research worker has his own way of expressing the pressure drop, while each material conveyed by air has its own equation on the pressure drop.

It will not do to design a pneumatic conveyor system for fiber assemblies with the aid of equations on the pressure drop for grain transportation. We must design the conveyor system for fiber assemblies by drawing on equations on the pressure drop for fiber assembly transportation. A conveyor system for fiber assemblies being generally composed of combinations of horizontal and straight conveyor pipes and bent conveyor pipes, we must calculate the total pressure drop for this system in designing it. The present article tries to clarify the relations between the mixing ratio and the pressure drop concerning horizontal and vertical straight conveyor pipes and bent conveyor pipes.

\section{Symbols}

$A_{1}, A_{2}$ : Cross section area at the entrance to and exit of bent pipe $\left(\mathrm{m}^{2}\right)$

$C$ : Acoustic velocity in fluid flowing in bent pipe $(\mathrm{m} / \mathrm{s})$

D: $\quad 4 \times \mathrm{D}_{m}(\mathrm{~m})$

$D_{m}: \quad$ Hydraulic mean depth (m)

$F_{r}: \quad$ Froude's number

$g$ : Gravitational acceleration $\left(\mathrm{m} / \mathrm{s}^{2}\right)$

$H$ : $\quad$ A side of cross section of duct (m) 
$H / W:$ Aspect ratio of duct

$h$ : $\quad$ Fluid friction loss of head or length of vertical pipe (m)

$L_{d}: \quad$ Downstream length of bent pipe (m)

$L_{u}$ : $\quad$ Upper stream length of bent pipe (m)

$l: \quad$ Length of pipe $(\mathrm{m})$

$l ' \quad \quad$ Length of center line of bent pipe (m)

$M$ : $\quad$ Mach's number

$m: \quad$ Mixing ratio (weight mixing ratio)

$\Delta p: \quad$ Pressure loss $\left(\mathrm{kg} / \mathrm{m}^{2}\right)$

$\Delta p_{b}$ : Pressure loss in bent pipe $\left(\mathrm{kg} / \mathrm{m}^{2}\right)$

$\Delta p_{b a}$ : Pressure loss in bent pipe when air is flowing $\left(\mathrm{kg} / \mathrm{m}^{2}\right)$

$\Delta p_{b f}$ : Additional pressure loss in bent pipe caused by fiber assembly $\left(\mathrm{kg} / \mathrm{m}^{2}\right)$

$\Delta p_{d}$ : Pressure loss in lower stream uninfluenced by bend $\left(\mathrm{kg} / \mathrm{m}^{2}\right)$

$\Delta p_{u}$ : $\quad$ Pressure loss in upper stream uninfluenced by bend $\left(\mathrm{kg} / \mathrm{m}^{2}\right)$

$R$ : $\quad$ Radius of curvature of center line of bent pipe (m)

$R_{e}: \quad$ Reynolds' number

$R / W: \quad$ Radius ratio

$u$ : $\quad$ Mean velocity in pipe $(\mathrm{m} / \mathrm{s})$

$u_{f}: \quad$ Friction velocity $(\mathrm{m} / \mathrm{s})$

$W: \quad$ A side of cross section of pipe (m)

$\gamma: \quad$ Specific weight of air $\left(\mathrm{kg} / \mathrm{m}^{3}\right)$

$\varepsilon: \quad$ Roughness of surface $(\mathrm{m})$

$\varepsilon / D: \quad$ Relative roughness

5 : $\quad$ Coefficient of additional pressure loss in bent pipe caused by fiber assembly

$\theta: \quad$ Angle of bend

$\Lambda$ : $\quad$ Coefficient of fluid friction on mixed-phase flow of air and fiber assembly in horizontal pipe

$\Lambda^{\prime}$ : Coefficient of fluid friction on mixed-phase flow of air and fiber assembly in vertical pipe

$\lambda: \quad$ Coefficient of fluid friction

$\lambda_{f}$ : Additional coefficient of fluid friction by fiber assembly in horizontal pipe

$\lambda_{f}^{\prime}: \quad$ Additional coefficient of fluid friction coming

from presence of fiber assembly in vertical pipe

$\mu$ : $\quad$ Coefficient of viscosity of fluid $\left(\mathrm{kg} \cdot \mathrm{s} / \mathrm{m}^{2}\right)$

$\xi: \quad$ Coefficient of pressure loss in bent pipe

$\rho: \quad$ Specific mass of air $\left(\mathrm{kg} \cdot \mathrm{s}^{2} / \mathrm{m}^{4}\right)$

$\tau_{0}: \quad$ Frictional stress on wall surface $\left(\mathrm{kg} / \mathrm{m}^{2}\right)$

\section{Experimental Apparatus}

An outline of our experimental apparatus is as shown in Fig.1. In our experiment we used the vacuum pneumatic conveyor system. We first weighed an assembly of fibers obtained from cotton lap and put it on belt-conveyor (1). We then broke the fiber assembly into pieces about $10 \mathrm{~mm}$ to $40 \mathrm{~mm}$ in diameter with a small special scratcher. The pieces were drawn into duct (3) which had a $50 \mathrm{~mm}$ square

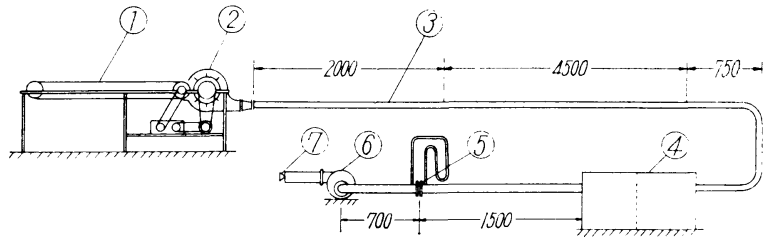

Fig. 1 Experimental apparatus

cross section made of sheet zinc. The duct had holes $(1 \mathrm{~mm}$ in diameter) at intervals of $500 \mathrm{~mm}$ on every side for the measurement of pressure.

After passing through duct (3), the fiber assembly was separated from the air in box (4). (5) was an orifice for the measurement of the air flow rate. The mean velocity of the air flowing through the duct was measured by this orifice. (6) was a turbo-blower. The air flow rate was controlled by (7).

\section{Pressure Loss in Horizontal Straight Pipes}

In dealing with the pressure loss in flow through a duct, we should not ignore the roughness of the inner surface of the duct. If the roughness of the inner surface of a duct varies, the pressure loss, when we let the air flow, varies and so also does the additional pressure loss coming from the presence of a fiber assembly. Therefore, to grasp the pressure loss in a pneumatic conveyor for fiber assemblies, we had to clarify the coefficient of fluid friction when only the air flowed through the pipes used in our experiment.

Those pipes had square cross sections and the flow of the air belonged to the domain of turbulence. Accordingly, to calculate the frictional loss of head, we used $4 \mathrm{Dm}$ as the representative scale showing the size of the cross section, rather than the diameter of a circular pipe. Frictional loss $h$ of head can be expressed thus:

$$
h=\lambda \frac{l}{4 D_{m}}-\frac{u^{2}}{2 g}
$$

the hydraulic mean depth ${ }^{[2]} \mathrm{Dm}$ being defined by

$$
D_{m}=\frac{\text { Cross section area of a pipe }}{\text { Peripheral length of cross section of pipe }}
$$

When only the air flows, $\lambda$ is obtainable, as in Fig. 2 , from the experimental data using eq.(1). Fig. 2 also shows Blasius' equation $^{[2]}$ as applied within the range of Raynolds' number $3 \times 10^{3}-10^{5}$ for smooth circular pipes.

$$
\lambda=\begin{gathered}
0.3164 \\
\operatorname{Re} !
\end{gathered}
$$

With the aid of pressure loss $\Delta p$, we can obtain the frictional stress on the surface of a wall. From this frictional stress we can obtain the friction velocity ${ }^{[1]} u_{f}$ during conveyance of a fiber assembly.

The flow in horizontal straight pipes within the limits of this experiment belongs to the domain of turbulence. Therefore, by using the concept of equilibrium of force, we can get the relation between the frictional stress $\tau_{0}$ and 


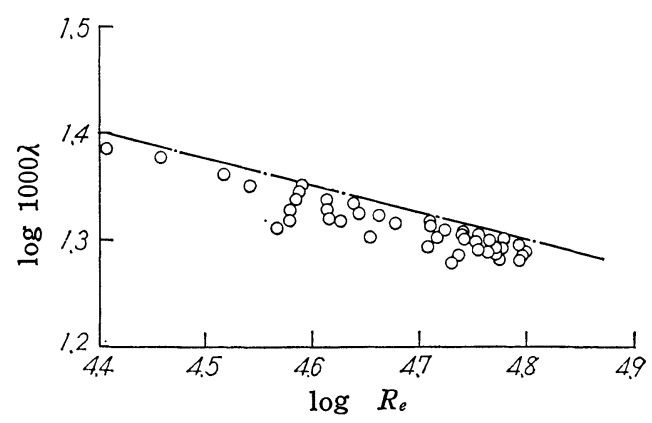

Fig. 2 Renolds' number and coefficient of fluid friction coefficient $\lambda$ of fluid friction, as follows:

$$
\frac{\pi}{4} D^{2} \Delta p=\pi D l \tau_{0}
$$

where $D=4 D_{m}$.

From the equation on energy, the following equation is obtainable:

$$
\Delta p=\lambda_{D}^{l} \frac{\rho u^{2}}{2} .
$$

Eqs. (3) and (4) lead to:

$$
\sqrt{\frac{\tau_{0}}{\rho}}=\sqrt{\frac{\lambda}{8}} \cdot u
$$

$\sqrt{\tau_{0} / \rho}$ has a dimension of velocity, which we will call the friction velocity and denote by $u_{f}$ :

$$
\sqrt{\frac{\tau_{0}}{\rho}}=u_{f}
$$

Substitute for $\sqrt{\tau_{0} / \rho}$ in eq. (5):

$$
\frac{u}{u_{f}}=\sqrt{\frac{8}{\lambda}}
$$

and eq. (3) reduces itself to

$$
\tau_{0}=-\frac{\Delta p}{l} \quad \frac{D}{4}
$$

Therefore, with values of $\Delta p$ experimentally obtained, values of $\tau_{0}$ are obtainable by eq. (8). Using this $\tau_{0}$, we get values of friction velocity $u_{f}$ from eq. (6). The relation between $u / u_{f}$ and $m$ on the basis of our experimental data

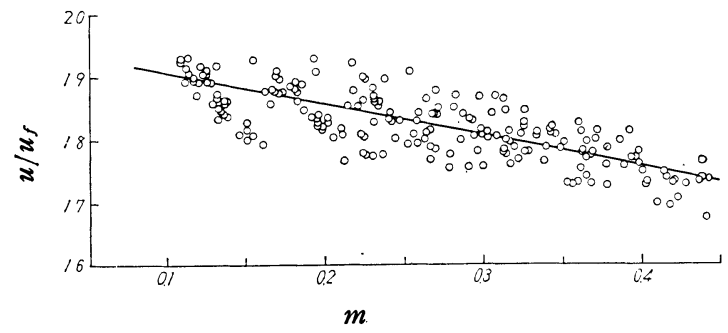

Fig. 3 Relation between $u / u_{f}$ and $m$ in horizontal straight pipe is as shown in Fig.3. The relation between $u / u_{f}$ and $m$, then, is expressible by a straight line. When no fiber assembly is in the pipe, the mixing ratio $m$ is zero and $u / u_{f}$ is $\sqrt{8 / \lambda}$ at $m=0$, in the light of eq. (7). Hence:

$$
\frac{u}{u_{f}}=\sqrt{\frac{8}{\lambda}}-5.10 \mathrm{~m}
$$

Assuming that $\lambda_{f}$ is the additional coefficient of fluid friction coming from the presence of a fiber assembly, then

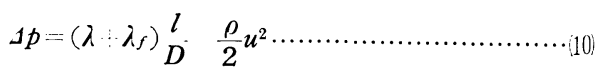

From Eqs. (8) and (10), the following equation is obtainable:

$$
\tau_{0}=\frac{\lambda}{8} \rho u^{2}\left(1+\frac{\lambda_{f}}{\lambda}\right)
$$

Therefore,

$$
\frac{u_{f}}{u}=\sqrt{\frac{\lambda}{8}\left(1+\frac{\lambda_{f}}{\lambda}\right) \cdots \cdots \cdots \cdots \cdots \cdots \cdots \cdots \cdots \cdots \cdots(12)}
$$

Eqs. (9) and (12) lead to:

$$
\lambda_{f}=\frac{8}{\left(\sqrt{\frac{8}{\lambda}}-5.10 m\right)^{2}-\lambda \ldots \ldots \ldots \ldots \ldots \ldots \ldots \ldots \ldots \ldots \ldots \ldots \ldots}
$$

this being the equation on additional pressure loss coming from conveying a fiber assembly. Hence, $\lambda_{f}$ is obtainable from coefficient $\lambda$ of fluid friction and mixing ratio $m$.

Assume that $\Lambda$ is the coefficient of fluid friction for mixedphase flow:

$$
A=\lambda+\lambda_{f} \quad \cdots \cdots \cdots \cdots \cdots \cdots \cdots \cdots \cdots \cdots \cdots \cdots \cdots \cdots \cdots \cdots \cdots \cdots \cdots \cdots
$$

Therefore, eqs. (13) and (14) lead to:

$$
A=\frac{8}{\left(\sqrt{\left.\frac{8}{\lambda}-5.10 m\right)^{2}}\right.}
$$

\section{Pressure Loss in Vertical Straight Pipes}

The pressure loss in vertical straight pipes is composed of the pressure loss in horizontal straight pipes and the pressure loss caused by gravity. The relation between $u / u_{f}$ and $m$ is as shown in Fig. 4 on the basis of experimental data.

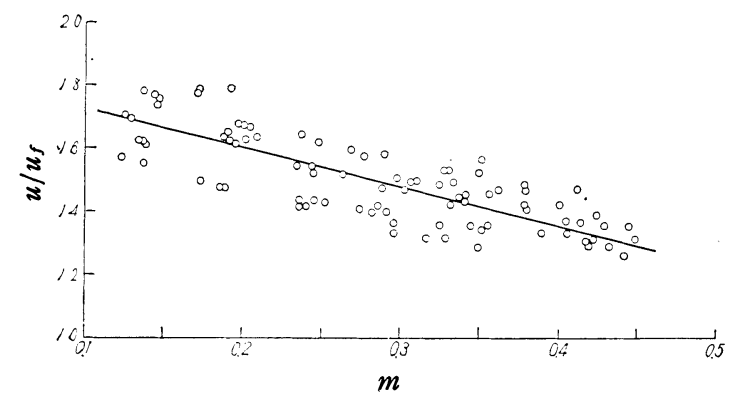

Fig. 4 Relation between $u / u_{f}$ and $m$ in vertical straight pipe 
Let the air flow in vertical pipes and assume that $\Delta p$ is the difference in static pressure between points $A$ and $B$ which are spaced apart by height $h$. Then:

$$
\frac{\Delta p}{\gamma}=h+\lambda \frac{h}{D} \frac{u^{2}}{2 g} \cdots \cdots \cdots \cdots \cdots \cdots \cdots \cdots \cdots \cdots \cdots \cdots \cdots \cdots \cdots \cdots
$$

Assuming that the pressure loss due to the height is included in frictional stress $\tau_{0}$, then:

$$
\tau_{0}=\frac{\Delta p}{h} \frac{D}{4} \cdots \cdots \cdots \cdots \cdots \cdots \cdots \cdots \cdots \cdots \cdots \cdots \cdots \cdots \cdots \cdots \cdots \cdots \cdots
$$

The friction velocity is, therefore, expressible as follows:

$$
\left.u_{f}=\sqrt{\frac{\tau_{0}}{\rho}}=\sqrt{\frac{\Delta \rho}{h}} \frac{D}{4} \frac{g}{\gamma} \ldots \ldots \ldots \ldots \ldots \ldots \ldots \ldots \ldots \ldots \ldots \ldots \ldots \ldots\right)
$$

Hence:

$$
\begin{aligned}
& \tau_{0}=\frac{D}{4} \gamma\left(1+\lambda \frac{1}{D}-\frac{u^{2}}{2 g}\right)=\frac{u_{f}^{2} \gamma}{g} \ldots \ldots \ldots \ldots \cdots \cdots \cdots(19) \\
& \frac{u}{u_{f}}=\sqrt{\frac{8}{\lambda}\left(1-g \frac{D}{4} \frac{1}{u^{2}}\right)} \cdots \cdots \cdots \cdots \cdots \cdots \cdots \cdots \cdots \cdots \cdots,
\end{aligned}
$$

When only the air flows, pressure loss $\Delta p$ is $10 \mathrm{mmAq}$ at $u=15.1 \mathrm{~m} / \mathrm{s}$, so that $u_{f}^{2}$ is 1.05 in the light of eq. (18). Eq.(20), then, transforms into:

$$
\frac{u}{u_{f}}=\sqrt{\frac{8}{\lambda}} \times \sqrt{1-0.116}=0.9597 \sqrt{\frac{8}{\lambda}} \cdots \cdots \cdots \cdots \cdots 211
$$

which is applicable where only the air flows.

The relation between $u / u_{f}$ and $m$ in mixed-phase flow made up of the air and a fiber assembly is expressible by a straight line as shown in Fig.4. Hence the following equation forms :

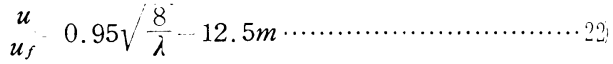

When only the air flows, the influence of height, i.e., factors $\sqrt{8 / \lambda}$ in eqs. (21) and (22), agree with each other, to wit:

$$
\begin{aligned}
& \left.\Delta p=\left(\lambda+\lambda_{f}^{\prime}\right) \frac{h}{D} \cdot \frac{\rho}{2} u^{2} \quad \ldots \ldots \ldots \ldots \ldots \ldots \ldots \ldots \ldots, 2\right), \\
& \tau_{0}=\frac{\Delta p}{h} \frac{D}{4}
\end{aligned}
$$

Accordingly:

$$
\begin{aligned}
& \tau_{0}=\frac{\lambda}{8} \rho u^{2}\left(1+\frac{\lambda_{f}^{\prime}}{\lambda}\right) \quad \cdots \cdots \ldots \ldots \ldots \ldots \ldots \ldots \ldots \ldots \ldots \ldots \\
& \left.u_{f}=\sqrt{\frac{\lambda}{8}\left(1+\frac{\lambda_{f}^{\prime}}{\lambda}\right)} \ldots \ldots \ldots \ldots \ldots \ldots \ldots \ldots \ldots \ldots \ldots+26\right)
\end{aligned}
$$

From eqs.(22) and (25) the following equation is deducible:

$$
\lambda_{f}^{\prime}=\frac{8}{\left(0.95 \sqrt{\left.\frac{8}{\lambda}-12.5 m\right)^{2}}\right.}-\lambda \ldots \ldots \ldots \ldots \ldots \ldots \ldots(27)
$$

We have divided the pressure loss in vertical straight pipes for the pneumatic conveyance of fiber assemblies into the pressure loss only in the air in horizontal straight pipes and the pressure loss added by fiber assemblies in vertical straight pipes. We have found that the latter is expressible by eq.(27), the $\lambda_{f}^{\prime}$ being calculated from the coefficient of fluid friction of the air and mixing ratio $m$.

Assuming $\Lambda^{\prime}$ to be coefficient of fluid friction of the mixed-phase flow of the air and fiber assemblies in vertical straight pipes, then:

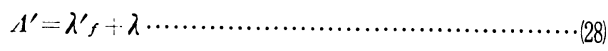

so that

$$
A^{\prime}=\frac{8}{\left(0.95 \sqrt{\left.\frac{8}{\lambda}-12.5 m\right)^{2}} \cdots \cdots \cdots \cdots \cdots \cdots \cdots \cdots(29)\right.}
$$

\section{Pressure Loss in Bent Pipes}

(1) Defining pressure loss and coefficient of loss in bent pipes

Generally, the pressure loss in bent pipes is expressible as follows ${ }^{[3]}$.

$$
\begin{aligned}
& \Delta p=F(u, c, \rho, \mu, g, D, R, H, W, \varepsilon, \\
& \left.l^{\prime}, A_{1}, A_{2}\right) \ldots \ldots \ldots \ldots \ldots \ldots \ldots \ldots \ldots \ldots \ldots \ldots(30)
\end{aligned}
$$

Express this equation in dimensionless numbers by the method of dimensional analysis. Since $u$ and $c, D, R, H$, $W, \varepsilon$ and $l, A_{1}$ and $A_{2}$ all have the same dimension, and in view of

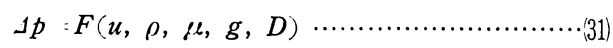

the following dimensional matrix is obtainable:

$$
\begin{aligned}
& \begin{array}{llllll}
u & \rho & \& & \Delta p & g & D
\end{array} \\
& \begin{array}{l}
L \\
T \\
T
\end{array}\left(\begin{array}{rrrrrr}
1 & -4 & -2 & -2 & 1 & 1 \\
0 & 1 & 1 & 1 & 0 & 0 \\
-1 & 2 & .1 & 0 & -2 & 0
\end{array}\right)
\end{aligned}
$$

As this matrix has rank 3, dimensionless products are 3 in number:

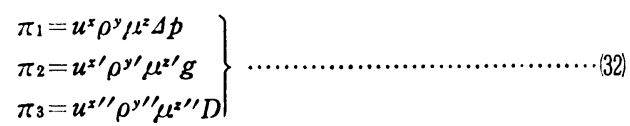

The values of $x, y, z, x^{\prime}, y^{\prime}, z^{\prime}, x^{\prime \prime}, y^{\prime \prime}$ and $z^{\prime \prime}$ calculated with the aid of the foregoing equation are:

$$
\begin{array}{lll}
x=-2, & x^{\prime}=-3, & x^{\prime \prime}=1 \\
y=-1, & y^{\prime}=-1, & y^{\prime \prime}=1 \\
z=0, & z^{\prime}=1, & z^{\prime \prime}=-1
\end{array}
$$

Hence:

$$
\left.\begin{array}{l}
\pi_{1}=\frac{\Delta p}{\rho u^{2}} \\
\pi_{2}=\frac{g / \ell}{u^{3} \rho} \\
\pi_{3}=\frac{D u \rho}{\mu}
\end{array}\right\}
$$

Using dimentionless product $F_{r}{ }^{-1}$ in place of $\pi 2$ results in

$$
\begin{aligned}
& \frac{\Delta p}{\frac{\rho u^{2}}{2}}=f\left(\frac{\rho u D}{\mu}, \frac{u}{\sqrt{g D}}, \frac{u}{c}, \begin{array}{r}
R \\
W
\end{array}, \frac{H}{W}, \frac{\varepsilon}{D},\right.
\end{aligned}
$$

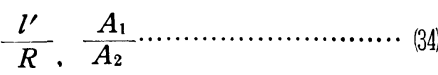


where

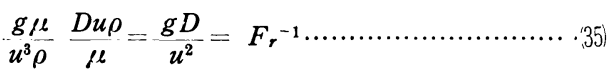

or

$$
\xi=f\left(R_{e}, F_{r}, M, \frac{R}{W}, \underset{W}{H}, \frac{\varepsilon}{D}, \theta, \frac{A_{1}}{A_{2}}\right) \cdots \cdots(36)
$$

Since we are dealing here only with bent pipes having a constant and square cross section area and a bent angle of $90^{\circ}$, we may omit the terms of $A_{1} / A_{2}, \theta$, and $H / W$. Since we may ignore compressibility at the velocity of a pneumatic conveyor, we may also omit Mach's number $M$.

Froude's number $\mathrm{Fr}$ is a number showing the influence of gravity. Since only horizontal bent pipes are considered here, we may ignore the influence of gravity and omit Froude's number. $\varepsilon / D$ and $R e$ are replaceable with coefficient $\lambda$ of fluid friction in straight pipes. Hence, the coefficient of the pressure loss in bent pipes is a function of $\lambda$ and $R / W$, i.e., $\lambda$ and $R / D$.

$$
\xi=f\left(\lambda, \frac{R}{W}\right)
$$

The $R / D$ of the bent pipes used in this experiment are as given in the following table:

Table 1 Measurements of Bent Pipes Used in Our Experiment

\begin{tabular}{cllc}
\hline Angle of bend & D mm & R mm & R/D \\
\hline 90 & 50 & 500 & 10 \\
90 & 50 & 400 & 8 \\
90 & 50 & 300 & 6 \\
90 & 50 & 200 & 4 \\
\hline
\end{tabular}

The flow of air being deformed noticeably at the exit of bent pipes, a fairly long length is needed for the gradual recovery of the velocity distribution, which is deformed, and for the full re-development of flow. It is difficult to measure the pressure drop near the inlet or outlet of pipes exactly, because it is affected by the curve of the pipes. We, therefore, measured the pressure drop at the point of upper flow $L_{u} / D=40$ and lower flow $L_{d} / D=30$.

Fig. 5 denotes the length of upper flow $\mathrm{AB}$ in the straight section of a bent pipe by $L_{u}$ and that of lower flow $C D$ in the bent section by $L_{d}$. Pressure losses in straight sections $A B$ and $C D$ without the influence of the bent section $B C$ are denoted by $\Delta \boldsymbol{P}_{u}$ and $\Delta \boldsymbol{P}_{d}$; the pressure loss in $A D$, by $\Delta p$. The pressure loss in bent section $B C$ is definable as follows :

$$
\Delta p_{b}=\Delta p-\Delta p_{u}-\Delta p_{d} \cdots \cdots \cdots \cdots \cdots \cdots \cdots \cdots \cdots \cdots \cdots \cdots \cdots(38)
$$

Denote the pressure loss when only the air flows in pipes by the suffix $a$; and the additional pressure loss caused by

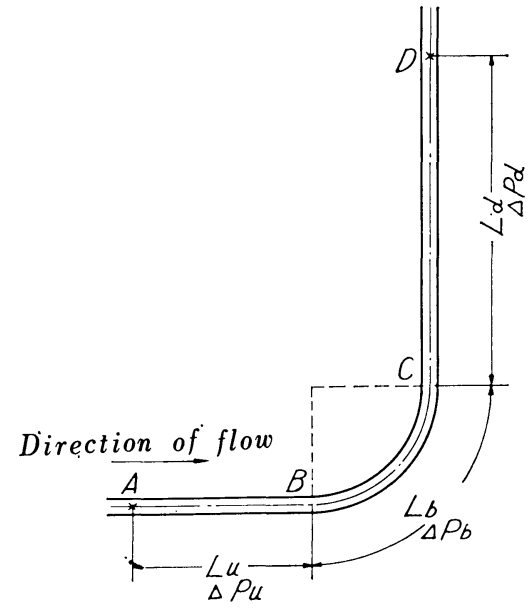

Fig. 5 Pressure loss in bent pipe

a fiber assembly by the suffix $f . \Delta p_{b}$, then, takes this form:

$$
\Delta p_{b}=\Delta p_{b a}+\Delta p_{b f} \cdots \cdots \cdots \cdots \cdots \cdots \cdots \cdots \cdots \cdots \cdots \cdots \cdots \cdots \cdots \cdots \cdots(39)
$$

Assuming $\Delta p$ to be the pressure loss between points $A$ and $B$ at mean air velocity $u$, then coefficient $\xi$ of the pressure loss in bent pipes and additional coefficient $\zeta$ of the pressure loss in bent pipes caused by a fiber assembly are definable by the following equations:

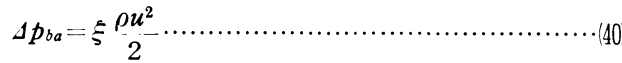

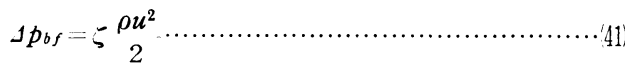

(2) Pressure loss in bent pipes

With $\Delta p_{u}, \Delta p_{b}$ and $\Delta p_{d}$ defined as in Fig.5, $\Delta p_{b}$ can be given by eqs.(39), (40) and (41).

The relations between coefficient $\xi$ of pressure loss and Reynolds' number $R e$ are as shown in Figs. 6 and 7.

Assume that $\Delta p_{b}$ is the pressure loss in mixed-phase flow in bent pipes. Divide it into the pressure loss $\Delta p_{b a}$ only in air flow and additional pressure loss $\Delta p_{b f}$ caused by a fiber assembly. By using this $\Delta p_{b f}$, we obtain additional coefficient $\zeta$ of pressure loss caused by a fiber assembly as in eq.(41). Examples of the relation between this coefficient $\zeta$ and the mixed ratio $m$ are as shown in Figs.8 and 9, from which is deducible the following equation:

$$
\left.\begin{array}{ll}
\zeta=20(0.104 m-0.0108) & \text { for } 2 R / D=20 \\
\zeta=16(0.114 m-0.0113) & \text { for } 2 R / D=16 \\
\zeta=12(0.112 m-0.0112) & \text { for } 2 R / D=12 \\
\zeta=8(0.109 m-0.0107) & \text { for } 2 R / D=8
\end{array}\right)
$$

which, consolidated, transforms into:

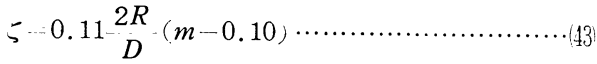

$R$ being the radius of curvature of the center line of a bent pipe; $D$ the hydraulic mean depth of a square pipe; and $m$ 


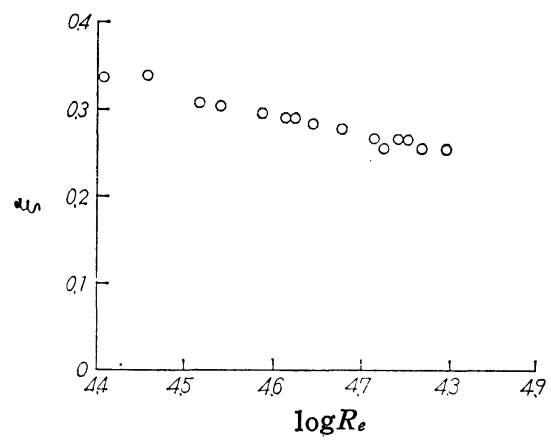

Fig. 6 Reynolds' number $R_{e}$ and coefficient $\xi$ of pressure loss $\left(2 R / D=20, \theta=90^{\circ}\right)$

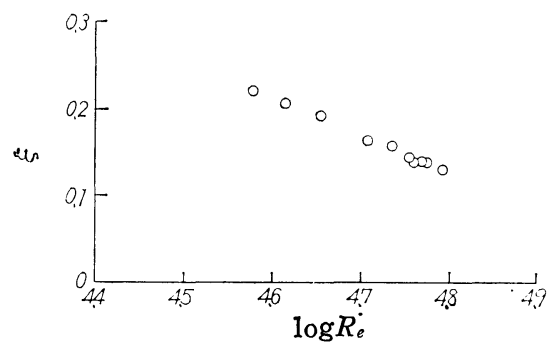

Fig. 7 Reynolds' number $R_{e}$ and coefficient $\xi$ of pressure loss $\left(2 R / D=12, \theta=90^{\circ}\right)$

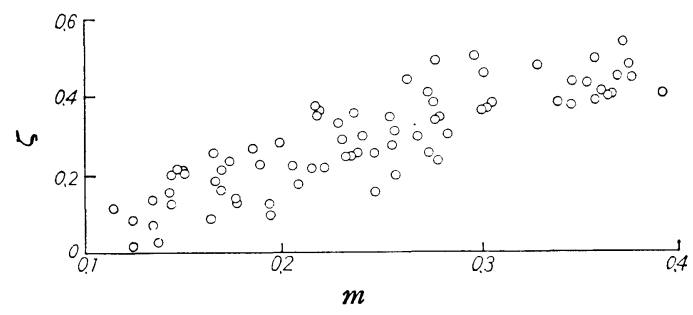

Fig. 8 Mixing ratio $m$ and coefficient $\zeta$ of pressure loss $\left(2 R / D=20, \theta=90^{\circ}\right)$

the mixing ratio.

The pressure loss in bent pipes needed in designing a pneumatic conveyor for fiber assemblies is obtainable

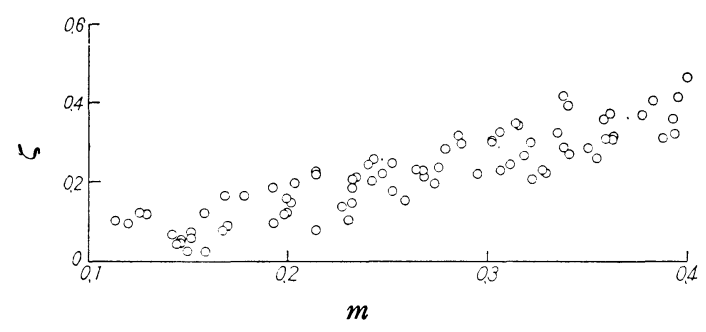

Fig. 9 Mixing ratio $m$ and coefficient $\zeta$ of pressure loss $\left(2 R / D=12, \theta=90^{\circ}\right)$

by eq. (44):

$$
\Delta p_{b}=(\xi+\zeta) \frac{\rho u^{2}}{2}
$$
$\cdot(44)$

where $\zeta$ is given as in eq.(43).

\section{Conclusions}

The authors have explained the relations between the pressure drop and the mixing ratio in horizontal and vertical straight pipes and bent pipes as a problem bearing on the pressure drop in the pneumatic conveyance of fiber assemblies.

We have obtained coefficient $\Lambda$ of fluid friction for mixedphase flow composed of the air and a fiber assembly in a horizontal straight pipe from eq.(15), and coefficient $\Lambda^{\prime}$ of fluid friction for mixed-phase flow composed of the air and a fiber assembly in a vertical straight pipe from eq.(29).

We have divided the coefficient of pressure loss in bent pipes into coefficient $\xi$ of only the air and additional coefficient $\zeta$ caused by a fiber assembly, $\zeta$ having been obtained from eq.(43). Hence pressure loss $\Delta p_{b}$ in bent pipes is as given by eq.(44).

Grateful acknowledgements are due to Mr. Norio Ishii, Mr. Katsuaki Isobe, Mr. Tohru Nagashima, Mr. Sukeji Harima and Mr. Sachio Kinoshita for their contributions to our experiment.

\section{References}

[1] K. Morikawa; Studies on pneumatic conveying for particles, A thesis for a degree (Osaka University, Japan)

[2] T. Uematsu; Suirikigaku, Sangyotosho, Japan (1962)

[3] D.W. Locklin; Energy Lossess in 90-Degree Duct Elbows 